Cardiology

Department, Queen

Mary Block, Old

Church Hospital,

Romford RM7 0BE,

UK

M U A Mustafa

J D Stephens

Cardiology

Department,

Hammersmith

Hospital, Du Cane

Road, London

W12 0NN, UK

C S R Baker

Correspondence to: Dr Mustafa.

Accepted for publication 9 March 1998

\title{
Spontaneously terminating ventricular fibrillation and asystole induced by silent ischaemia causing recurrent syncope
}

\author{
M U A Mustafa, C S R Baker, J D Stephens
}

\begin{abstract}
A 57 year old man was admitted for investigation of recurrent syncopal attacks. Holter monitoring during an attack while in hospital revealed a unique sequence of gross ST segment elevation, ventricular tachycardia, prolonged ventricular fibrillation, asystole, junctional and ventricular escape rhythm, and finally spontaneous restoration of sinus rhythm with severe ST segment depression. Subsequent coronary arteriography demonstrated severe stenoses of the right coronary artery, prompt-
\end{abstract}

ing percutaneous transluminal angioplasty and stenting. The patient has had no further syncopal attacks.

(Heart 1998;80:86-88)

Keywords: ventricular fibrillation; Holter monitoring; coronary stenoses; silent ischaemia; arrhythmias

A 57 year old man presented to the accident and emergency department following a syncopal attack. There was no loss of bladder control, tongue biting or seizure. There was no

A

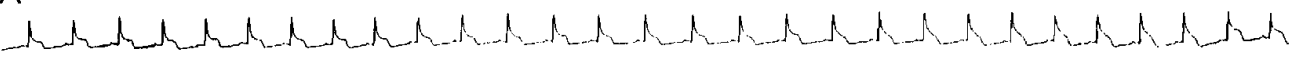

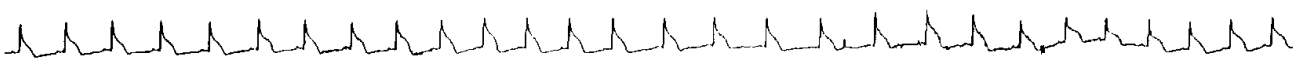

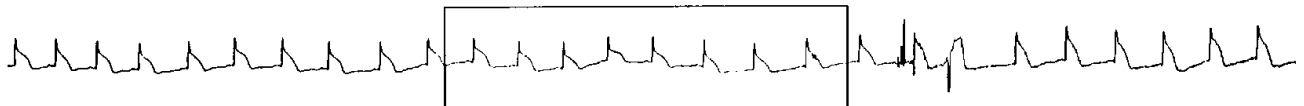

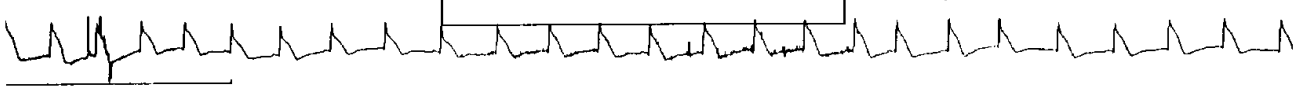

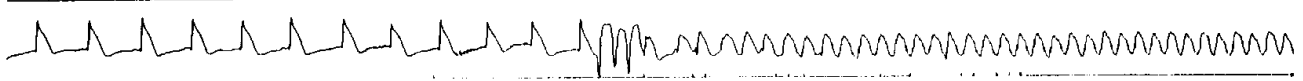
B

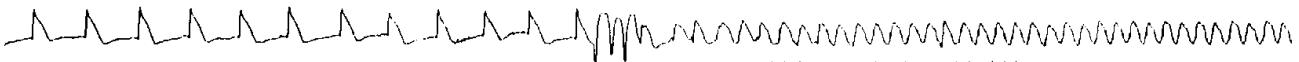

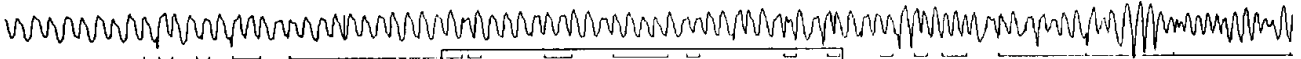

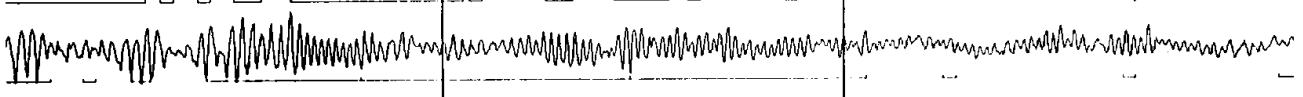

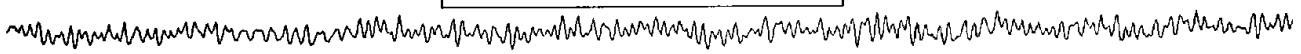

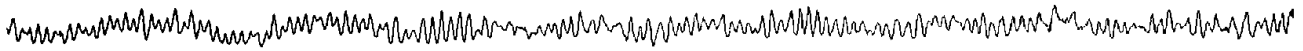
C

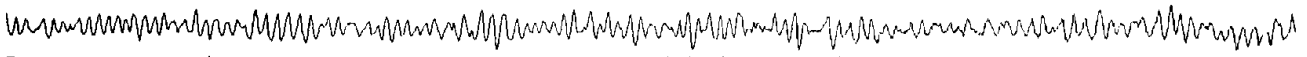

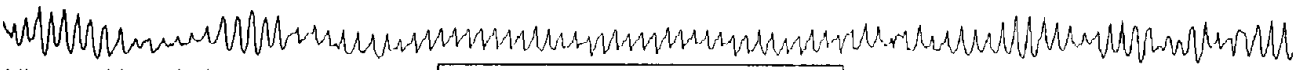

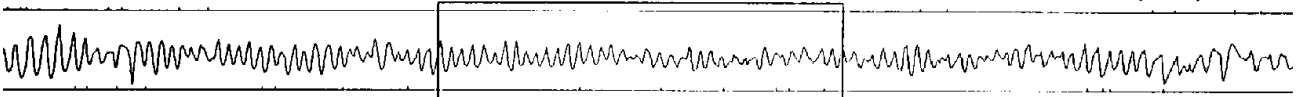
sumblor 
history of angina-like chest pain. A complete physical examination and initial routine investigations were normal. There was no medical history of any significance. There was a positive family history of ischaemic heart disease, two of his uncles having died from ischaemic heart disease. There was no history of sudden death in the family. The patient smoked $80-90 \mathrm{~g}$ of tobacco per week.

On examination he was well and had no fever. His pulse was 66 beats/min and regular,

D

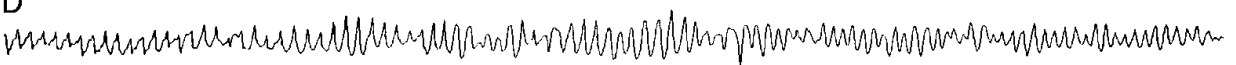

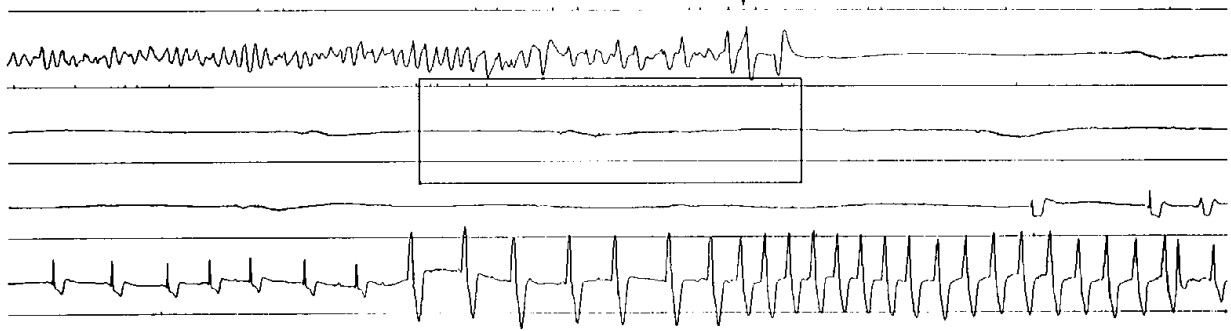

$E$

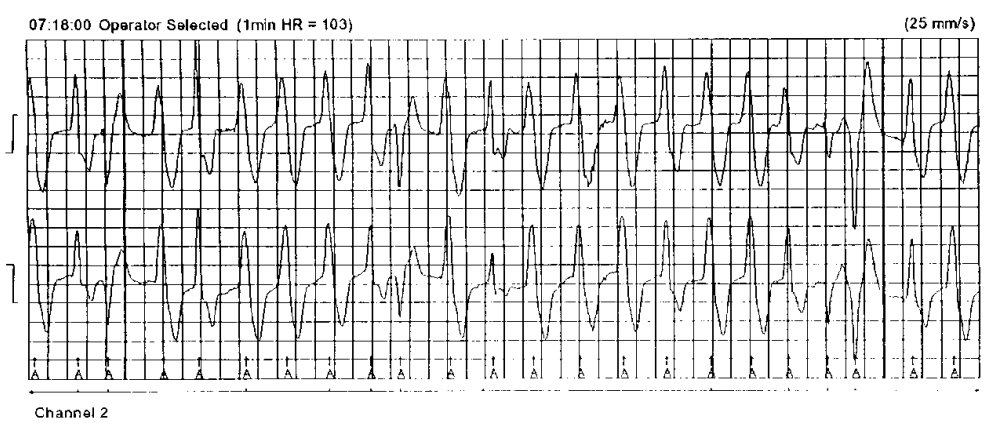

blood pressure was 130/75 mm Hg. Cardiovascular, respiratory, gastrointestinal, and central nervous system examinations were all normal. A routine 12 lead ECG and chest radiography were normal. All laboratory investigations including three sets of cardiac enzymes were normal.

The patient underwent 24 hour Holter monitoring during which he had an episode of chest tightness followed by loss of consciousness on the ward but regained consciousness
Figure 124 hour Holter monitor recording showing (A) onset of severe $S T$ segment elevation;

(B) ventricular tachycardia; (C) ventricular fibrillation; (D) ventricular asystole;

(E) junctional and

ventricular escape rhythm and $(F)$ sinus rhythm.

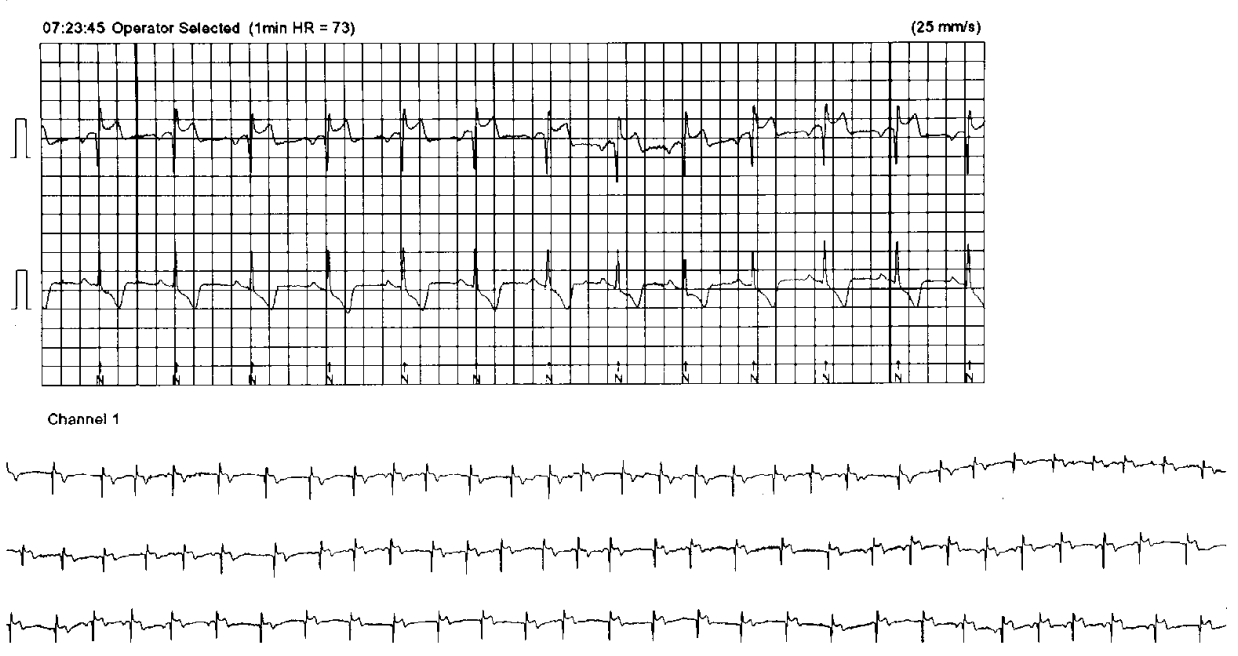

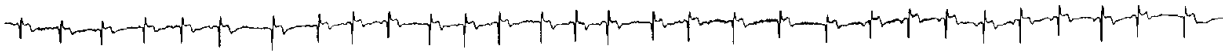

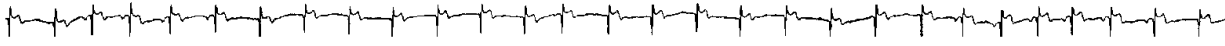



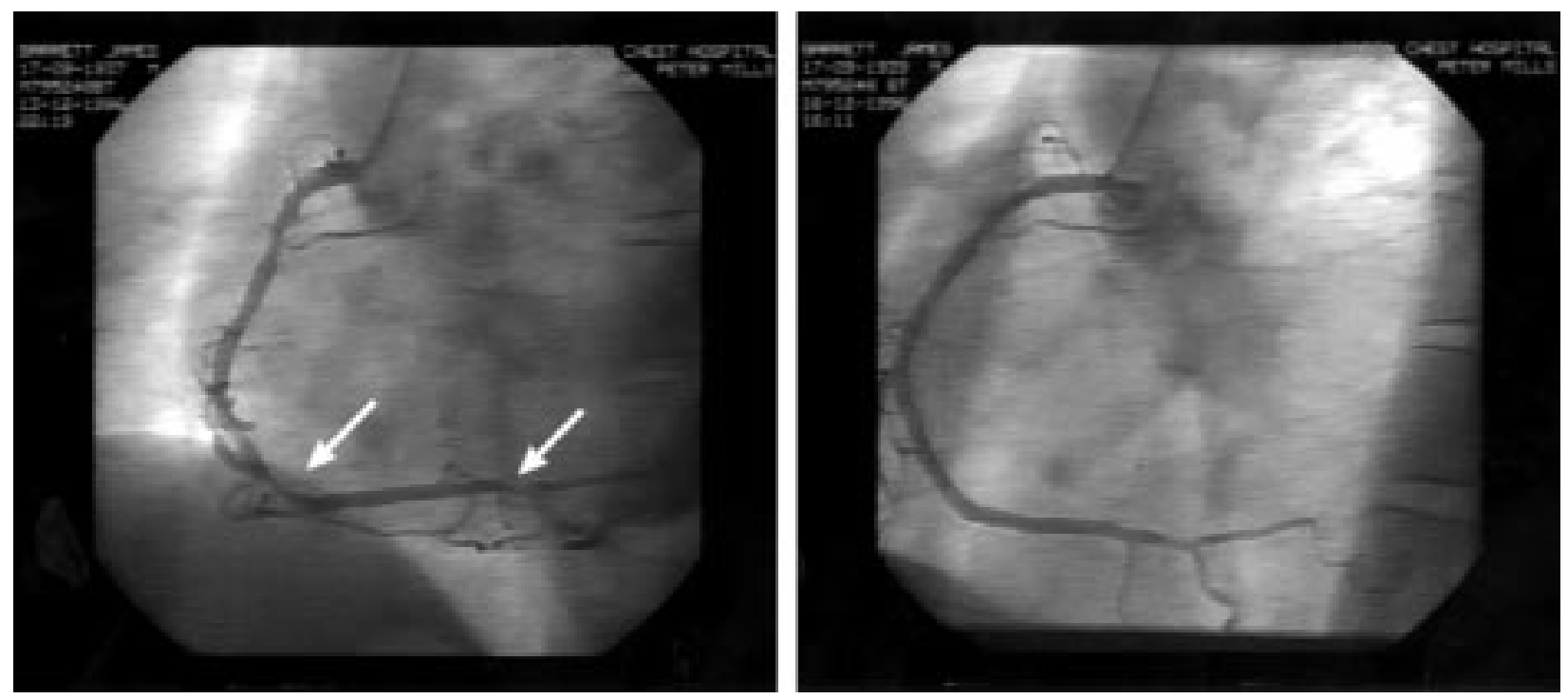

Figure 2 Left: Angiogram showing stenoses of the right coronary artery. Right: Same coronary artery after stenting.

before the cardiac arrest team arrived. An ECG on recovery showed sinus rhythm with inferolateral ST segment depression. The Holter recording showed several episodes of transient ST segment elevation before syncopal attack. Coinciding with the attack, there was a striking sequence of events - sustained ventricular tachycardia initiated by an $\mathrm{R}$ on $\mathrm{T}$ ventricular extrasystole, prolonged ventricular fibrillation (three minutes), prolonged asystole (63 seconds), AV block, slow and fast ventricular escape rhythm, junctional escape rhythm, and finally sinus rhythm with severe ST segment depression (fig 1). The whole episode from the onset of ventricular tachycardia to the spontaneous restoration of sinus rhythm lasted for eight and a half minutes. There was no rise in cardiac enzymes and there was complete resolution of the ST segment changes on the ECG.

Subsequent coronary arteriography revealed two severe stenoses of the right coronary artery (fig 2), which prompted percutaneous transluminal coronary angioplasty and stenting. The patient has had no further syncopal episodes.

\section{Discussion}

Spontaneously terminating ventricular fibrillation is rare but has been described before. ${ }^{1-4}$ Two cases where coronary arteriography was performed showed normal coronary arteries. ${ }^{12}$
One case had ventricular fibrillation followed by ST segment depression and was thought to be caused by coronary reperfusion following silent ischaemia.

Our case illustrates a unique combination of spontaneously terminating prolonged ventricular fibrillation and asystole triggered by silent ischaemia associated with severe coronary stenoses, successfully treated by PTCA and stenting.

Recurrent syncope is a common clinical problem with a variety of causes but serious ventricular arrhythmias caused by silent ischaemia should be considered in such patients. This case draws attention to the potential arrythmogenicity of silent ischaemia.

We are grateful to Dr Peter Mills and Dr Anthony Nathan, consultant cardiologists at Royal Hospitals NHS Trust for their invaluable help in presentation of this case report.

1 Van Hemel NM, Kingma JH. A patient in whom self-terminating ventricular fibrillation was a manifestation of myocardial reperfusion. Br Heart f 1993;69:568-71.

2 Masrani K, Cowley C, Bekhit S, et al. Recurrent syncope for over a decade due to idiopathic ventricular fibrillation. Chest 1994;106:1601-3.

3 Shaw TR. Recurrent ventricular fibrillation associated with normal QT intervals. $Q \mathcal{F}$ Med 1981;50:451-62.

4 Tye $\mathrm{KH}$, Desser K, Benchimol A. Survival following spontaneous ventricular flutter-fibrillation associated with QT syndrome. Documentation of ambulatory monitoring. Arch Intern Med 1980;140:255-6. 